\title{
Hindouisme et pratiques spatiales des Tamouls en Île-de-France
}

Hinduism and Spatial Practices of Tamils in Île-de-France

\section{Anthony Goreau Ponceaud}

\section{(2) OpenEdition}

\section{Journals}

Édition électronique

URL : https://journals.openedition.org/cdg/1405

DOI : $10.4000 /$ cdg. 1405

ISSN : 2107-7266

Éditeur

UMR 245 - CESSMA

Référence électronique

Anthony Goreau Ponceaud, « Hindouisme et pratiques spatiales des Tamouls en Île-de-France », Carnets de géographes [En ligne], 11 | 2018, mis en ligne le 15 septembre 2018, consulté le 20 mai 2021. URL : http://journals.openedition.org/cdg/1405; DOI : https://doi.org/10.4000/cdg.1405

Ce document a été généré automatiquement le 20 mai 2021.

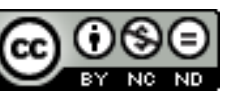

La revue Carnets de géographes est mise à disposition selon les termes de la Licence Creative Commons Attribution - Pas d'Utilisation Commerciale - Pas de Modification 4.0 International. 


\title{
Hindouisme et pratiques spatiales des Tamouls en Île-de-France
}

\author{
Hinduism and Spatial Practices of Tamils in Île-de-France
}

Anthony Goreau Ponceaud

\section{NOTE DE L'AUTEUR}

Ce texte est en partie issu d'une présentation menée lors d'un séminaire s'intégrant au programme « Dieu change à Paris. Les mutations religieuses dans l'agglomération parisienne » du GSRL (Groupe Sociétés, Religions, Laïcités) en octobre 2014. Je tiens à remercier les membres de ce séminaire et les responsables du programme, Martine Cohen et Sébastien Fath ainsi que les relecteurs de la revue Carnets de géographes pour leurs commentaires constructifs et judicieux. J'ai délibérément omis les diacritiques devanāgari des termes issus du Sanskrit présents dans le texte. Ce choix est justifié par une utilisation écrite similaire faite par les populations tamoules rencontrées sur le terrain.

\section{Introduction}

1 Le Sri Lanka, île de l'océan indien de plus de 20 millions d'habitants, dont les troisquarts ont pour langue maternelle le cinghalais (une langue issue du fonds indo-aryen) et le cinquième, le tamoul (langue dravidienne d'Inde du Sud), a été marqué par une guerre qui a opposé de juillet 1983 à mai 2009 l'armée sri-lankaise aux militants séparatistes tamouls des LTTE$^{1}$. Ce conflit a eu de larges répercussions dans les logiques migratoires des Sri Lankais: terre d'immigration depuis les débuts de son histoire, le Sri Lanka est devenu progressivement au cours des années soixante-dix, une terre d'émigration massive. La mise en place de lois discriminantes (Sinhala only Act faisant de la langue cingalaise la seule langue officielle au détriment des locuteurs de tamoul et donnant au bouddhisme une place prééminente dans l'État, tournant ainsi le dos à des 
décennies de sécularisme), a lancé sur les routes de l'exil, de manière sporadique dans un premier temps, des élites anglophones vers les pays du Commonwealth. Néanmoins, après les pogroms anti tamouls de juillet 1983, correspondant également à la montée en puissance des LTTE et à la substitution d'une idéologie de défense des droits des minorités par une idéologie de libération nationale, les flux de réfugiés et de demandeurs d'asile en direction des pays du Nord, d'Asie (Malaisie et Inde en particulier) et du Moyen-Orient sont devenus significatifs conduisant à la formation d'une diaspora ${ }^{2}$ et à une diversification des profils migratoires. Comme le notent Éric Meyer et Delon Madavan : «l'émigration sri-lankaise temporaire ou durable a concerné au cours de cette période $8 \%$ à $10 \%$ de la population de l'île, et les diasporas principalement tamoules représentent plus d'un million de personnes si l'on compte les descendants des migrants » $(2015: 220)$.

2 Depuis la décennie 1980, les réfugiés en provenance de Sri Lanka arrivent de manière régulière en France et constituent le plus grand groupe hindou. La grande majorité des Tamouls sri-lankais présents en France sont hindous (à plus de $80 \%$ ) mais il existe des minorités chrétiennes (entre 10 et $20 \%$ ) et musulmanes. Parmi les chrétiens la majorité est catholique, mais une minorité de plus en plus importante (d'un point de vue numérique) est protestante et plus spécifiquement pentecôtiste et évangélique. Alors que des communautés de langue tamoule sont accueillies au sein d'Eglises pentecôtistes locales, d'autres fondent des assemblées indépendantes (dont le nombre aujourd'hui n'est pas connu précisément - pas plus que le nombre de fidèles). La plus ancienne assemblée fondée en France par un missionnaire sri-lankais date des années 1950. Il s'agit de l'Eglise de pentecôte primitive, située à la Courneuve, créée par le pasteur Benjamin Selvaratnam qui a implanté en 1952 une œuvre missionnaire pour le compte de la Ceylon Pentecostal Mission (CPM), une des principales dénominations pentecôtistes de l'île.

3 Ces dernières années, cette migration, et plus généralement les migrations d'Asie du Sud, ont radicalement transformé le paysage social français, conduisant à ce que Grillo nomme un "excès d'altérité » (Grillo 2010), accentuant la pluralisation du paysage religieux et culturel ${ }^{3}$. Il s'agit dans cette contribution de questionner les dynamiques du changement urbain lié aux migrations et au fait religieux et de travailler la question de la religion dans la migration pour répondre à la question suivante: qu'est-ce qui change quand on change de place? Notons que ce changement de place peut générer des tensions dans les manières qu'a la société française de produire la croyance en ellemême et la ritualisation de cette croyance. En d'autres termes, la pluralisation du paysage religieux et culturel questionne la religion civile française, ce mélange subtil de France laïque et de France catholique qui contribue à sacraliser l'être-ensemble de la société française (Willaime, 2009). Plus largement, comme ont pu le noter Sophie Bava et Stefania Capone (2010), la circulation des migrants suscite de nouvelles dynamiques religieuses et en réactive d'anciennes. En effet, depuis la décennie 1990, en lien avec le champ d'étude transnational et la forte croissance des recherches menées sur les diasporas, on assiste à de nouveaux questionnements du lien entre migration et religion et du rôle des migrations dans la diffusion et la création religieuse. Il y a là un véritable engouement pour l'analyse des expressions des faits religieux dans les espaces urbains et leur globalisation ou transnationalisation (Dejean et Endelstein, 2013). Il faut dire que par leur nature même, les migrations reconfigurent l'offre religieuse (mais aussi commerciale) des grandes villes d'installation et le religieux est considéré comme 
un vecteur de recompositions identitaires pour des sujets pris dans une instabilité. Le religieux peut être un pôle structurant de l'organisation d'une communauté ${ }^{4}$ et support de territorialité : « l'existence de fondements à la présence sur un lieu permet d'ouvrir de nouveaux lieux dans des non-lieux, de l'unité dans l'émiettement " (Hovanessian, 2007, 12). Paris n'échappe pas à ce constat. Les Sri Lankais ont su se ménager une place dans l'espace urbain, une niche de visibilité, en constituant un quartier commercial à la Chapelle (pour en savoir plus sur les effets de ces dynamiques de placements voir Goreau-Ponceaud, 2018) et en créant leurs propres espaces de prière, soit en s'appuyant sur des réseaux religieux déjà existants (pour les catholiques chrétiens et pentecôtistes en particulier), soit en construisant de nouveaux espaces religieux (hindouisme singulièrement). Progressivement, les Sri Lankais ont mis en scène des territoires qu'ils connectent dans un espace transnational, décrivant les contours de territoires relationnels ${ }^{5}$. Ces connexions peuvent être à caractère économique, mais aussi culturel ou religieux dégageant progressivement les contours d'un savoir circuler, marge de manœuvre alimentant des imaginaires qui se situent entre un savoir-faire qui est le fruit de cette mobilité et un pouvoir-faire limitatif issu des contraintes exercées par la société française. A cette mise en forme d'une "translocalité " (Appadurai, 1995), ou d'un territoire relationnel, s'ajoute, pour la société d'accueil, une nouvelle dialectique invisibilité/visibilité dans l'espace urbain. En effet, si la présence de la religion peutêtre discrète et dérobée aux regards, on observe aussi le retour en force des processions, à l'instar de la procession de Ganesh ou Ganesh Caturthi qu'organise l'association Sri Manicka Vinayakar Alayam tous les ans dans les rues de Paris. En tant que tel, l'hindouisme ne représente pas seulement la tradition d'une minorité religieuse; il met également en tension des acteurs déjà ancrés dans la ville et des populations migrantes nouvellement arrivées (Goreau-Ponceaud, 2014). C'est donc à travers l'articulation entre immigration, religion et espace que je vais aborder l'enjeu $\mathrm{du}$ religieux, et plus spécifiquement l'enjeu du religieux en "mode mineur ${ }^{6}$ » (Piette, 1992) en ville. Entre local (la rue, le quartier) et global (flux d'immigration transnationaux ${ }^{7}$ ), c'est l'enjeu du rapport à l'espace qui constitue l'axe de cette réflexion où l'espace sera considéré comme un medium des rapports sociaux qui permet d'interroger de façon originale des recompositions du fait religieux aujourd'hui. Nous examinerons d'abord les effets de ce savoir-circuler tamoul sur le paysage urbain, avant d'appréhender la localisation des lieux de culte. Ce positionnement m'invitera à apporter une épaisseur temporelle, à considérer les communautés dans leur histoire et à les suivre dans leurs différents déplacements. Ainsi, je parlerai de parcours résidentiels pour désigner ce processus par lequel une communauté ne rentre pas forcément en adéquation avec un lieu de culte particulier mais connaît plusieurs localisations successives. Nous porterons aussi une attention particulière au processus - dénommé templeisation par Vasudhana Narayanan (1992) - par lequel les Tamouls hindous attribuent de nouvelles significations et une importance particulière au temple, dans un contexte diasporique; le temple permettant la mise en place d'un marquage territorial et l'inscription dans l'espace de la diaspora (Bauman, 2009).

\section{La Chapelle : un territoire relationnel}

4 Les trajectoires migratoires des Tamouls en France sont diverses et variées regroupant des communautés d'histoire différentes où la classe, la caste, la religion, le lieu d'origine et le statut viennent émietter les groupes (pour plus de détails sur ces 
trajectoires voir Goreau-Ponceaud, 2011). Cette immigration, majoritairement concentrée en région parisienne se caractérise par sa diversité interne qui s'accentue davantage encore sous l'effet des regroupements religieux, consécutifs tant à la circulation depuis le monde indien de certaines traditions religieuses (que l'on songe à l'hindouisme shivaïte ou au culte de la vierge de Velankanni ${ }^{8}$ notamment) qu'à l'émergence de nouveaux mouvements religieux de et en diaspora. Notons que même au sein de la diaspora tamoule en France, la distinction entre rites catholiques et rites hindous est par moments assez floue, car que ce soit l'hindouisme ou le catholicisme, ces deux religions en diaspora n'ont pas été transplantées outre-mer, mais transformées, si bien qu'il existe des syncrétismes qui complexifient l'analyse. Il n'est pas rare que catholiques et hindous fréquentent les mêmes lieux de culte et il existe une culture matérielle et des pratiques sensorielles partagées entre hindouisme et catholicisme. Comme avait déjà pu le noter Stine Bruland en 2013, on retrouve dans les deux cas des esthétiques similaires, à savoir l'utilisation de guirlandes de fleurs ou encore le mouvement de l'encens dans le sens des aiguilles d'une montre qui fait penser au rituel hindou d'āratī. Catholiques et hindous en Inde et à Sri Lanka ont intégré des croyances périphériques dans leurs rites, la migration n'a pas altéré ces syncrétismes mais les a intensifiées. Les enjeux démographique et symbolique de cette diversité sont considérables pour l'évolution du paysage socio-religieux en Île-de-France. Cependant, les phénomènes concernés sont encore très peu étudiés. Ces questions cruciales relatives à la transmission religieuse ont seulement été analysées principalement par rapport aux destinations classiques des réfugiés tamouls comme le Royaume-Uni, les États-Unis ou le Canada ${ }^{9}$. En conséquence, nous savons très peu de choses sur les spécificités de ces communautés les plus récentes et encore en croissance dans le contexte français.

\section{Une porte d'entrée sur l'espace national}

5 En premier lieu, il est indispensable de préciser que si les Tamouls sri-lankais ont fait le choix de s'installer en France, à partir de la fin des années 1970 et au début des années 1980, c'est par défaut. Leur choix se portait en priorité sur l'Angleterre, du fait notamment de la pratique de la langue anglaise, et d'une certaine "proximité " avec l'ancienne puissance coloniale. Cependant, à cause du durcissement des lois sur l'immigration (Immigration Act de 1971, visant à réduire l'immigration en provenance des pays du Commonwealth), les candidats à l'émigration en Angleterre ont dû stopper leur périple en France. C'est comme cela que l'on a assisté, entre les stations de métro la Chapelle et Gare du Nord, entre les $10^{\mathrm{eme}}$ et $18^{\mathrm{eme}}$ arrondissements de Paris, à la constitution progressive d'un entre-soi. Et si l'on tient compte de la morphologie du quartier, cet entre-soi a toutes les caractéristiques d'une porte d'entrée. Inséré entre deux gares (Gare de l'Est et Gare du Nord), limitrophe au Nord des quartiers immigrés d'implantations plus anciennes que sont Barbès, Château Rouge et la Goutte d'Or, offrant une importante concentration d'hôtels modestes et peu coûteux, ce quartier semble proposer, plus qu'ailleurs, des facilités pour s'établir seul, sans relais communautaire, du moins pour les primo-arrivants. 
Figure 1 : Le quartier La Chapelle

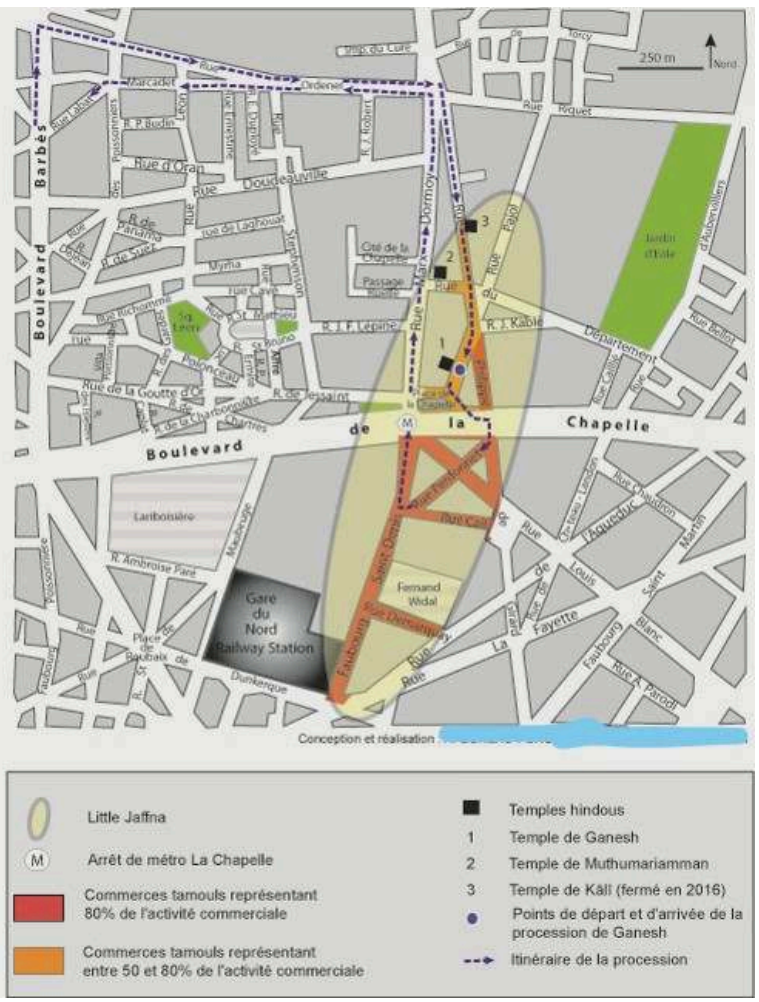

6 La Chapelle n'est pas seulement un " port » de première entrée. Ce quartier est aussi marqué par la concentration d'activités commerciales qui rend plus ou moins visible une différence culturelle dans l'espace urbain. En moins de trois décennies, la Chapelle est devenu une centralité commerciale minoritaire qui polarise une clientèle immigrée: ce sont les usagers qui donnent au lieu son image de "quartier sudasiatique ", et non les résidents. La Chapelle est souvent appréhendé comme un quartier sri-lankais, le Little Jaffna de Paris, du fait de sa spécialisation marchande qui a progressivement transformé le paysage commercial par la place croissante prise par les commerces dits ethniques, témoignant du dynamisme des activités et des entrepreneurs issus de ce courant migratoire. Ce quartier permet ainsi d'articuler l'analyse du changement urbain avec celle des pratiques sociales et culturelles de populations migrantes, dont les chaînes migratoires bien établies restent multiples et variées. Précisément, il s'avère que les fondateurs de ce commerce minoritaire sont les Marécars (ou Maraikayar). Ce sont des commerçants indiens (présents en grand nombre à Pondichéry et Karikal) musulmans qui arrivèrent directement dans les années 1960 du Vietnam en région parisienne. Ils furent les premiers à mettre en place des réseaux d'entraide communautaire à l'origine d'une véritable infrastructure commerçante sud-asiatique à Paris. À cela s'ajoute une présence importante de migrants d'Asie du Sud (Pakistanais, Bangladais, Gujaratis, Mauriciens) installés également depuis les années 1960 dans le $10^{\text {ème }}$ arrondissement, en particulier autour du Boulevard de Strasbourg, de la rue du Faubourg Saint-Denis (Passage Brady) et de la rue Jarry (Aly Marecar Vinay et Vuddamalay, 2007). Ainsi, ce serait pour des conditions de site et de situation, que les Tamouls sri-lankais se seraient implantés dans ce quartier. D'autres éléments peuvent expliquer cette concentration: les obligations financières liées au coût du voyage entre Sri Lanka et la France, la non-maîtrise de la langue française ou encore la mise en place de stratégies économiques pour acquérir 
des fonds de commerce à bas prix. De plus, ce commerce est souvent considéré par la communauté comme un moyen de contourner les obstacles d'un marché du travail discriminatoire, et joue un rôle important dans les stratégies de positionnement identitaire.

\section{Un territoire relationnel}

7 Ce théâtre monde, où s'exprime une complémentarité entre dynamiques locales et globales à travers des dispositifs marchands, est une scène privilégiée de la représentation que les Tamouls donnent d'eux-mêmes à la société d'accueil, mais aussi entre eux. Il est un moyen d'organiser la coprésence et la relation en des temporalités particulières. Ainsi, si les enseignes sont continuellement présentes et rappellent le référent origine, ce quartier connaît des temps de visibilité distincts qui s'expliquent par la dissociation entre lieu de vie et lieu de résidence. Le quartier la Chapelle est une centralité commerciale minoritaire qui ne l'est qu'au rez-de-chaussée! Cet exemple $\mathrm{d}^{\prime}$ ' écogenèse territoriale ${ }^{10}$ » qui fait appel à un processus de sémiotisation complexe (via des jeux d'enseigne et des stratégies d'appropriation) questionne les transformations territoriales induites par les migrations internationales. Plus exactement ce quartier est situé «au carrefour de plusieurs interactions entre le présent et le passé, le soi et l'autre, le local et le global» (Fourcade, 2008, 27). La Chapelle est un espace créé à la fois par les populations locales et les populations mobiles, un lieu où se mêlent les transactions économiques et les relations affectives. Cet exemple de translocalité (Appadurai, 1995) met l'accent sur toutes les formes de coprésence qui participent de la production des espaces pratiqués. Cette notion de translocalité exprime des espaces-temps articulés de réseaux sociaux qui associent les caractéristiques propres du lieu lui-même aux spécificités des liens qui le relient avec l'extérieur. De ce fait, ce sont ces liens, interpersonnels et inter-lieux qui participent pleinement de l'identité même de ce lieu et bousculent l'hypothèse du nationalisme méthodologique ${ }^{11}$. Ce paradigme de la translocalité, alimenté par un nouveau regard porté sur les mobilités permet d'indiquer qu'est «frontière » tout ce qui marque une asymétrie, maintient une tension et un différentiel qui conditionne les mouvements possibles et leur organisation. Les pratiques religieuses des Tamouls viennent alimenter cette translocalité. Le quartier offre en effet la particularité d'abriter trois temples hindous qui sont eux-mêmes pris dans des circulations multiples démontrant que les processus de déterritorialisation se font rarement sans une successive reterritorialisation.

\section{Les pratiques religieuses des tamouls hindous en Ile- de-France}

8 « Nous ne pouvons pas vivre dans un lieu où il n'y pas de dieux, c'est inconcevable. À notre arrivée, d'accord, il n'y avait pas de lieux de culte, pas de temples (kovil $\left.{ }^{12}\right)$, mais nous avions apporté avec nous les images de nos divinités. Elles étaient souvent froissées, abîmées, à cause du voyage, mais ce sont elles qui faisaient le lien entre ici et la maison. C'est grâce à elles que l'on pouvait exprimer notre foi. Autour de ces images on a créé des autels domestiques, une étagère sur laquelle trône ces images. D'ailleurs tous les Tamouls ont un autel domestique. Moi dessus il y a une image de Murugan, de 
Ganesh, de Muthumāriyamman et de l'eau que j'ai rapportée de Lourdes » (Balakrishnakurukal, fils du fondateur du temple de Murugan situé dans le 19 ème arrondissement près de l'arrêt de métro Jourdain, interrogé en 2008).

Pour les Sri Lankais la religion est un facteur important, non seulement parce qu'elle permet de maintenir des liens avec le pays d'origine, le homeland, mais aussi car elle permet d'orienter leur vie dans la diaspora.

\section{Transplanter les cultes hindous en France}

Alors que les premiers immigrants n'avaient pas de lieux où pratiquer l'hindouisme, au fil du temps, les Tamouls sri lankais ont créé des possibilités de pratiquer leur foi en dehors de leur maison. Je souhaiterais dans cette partie appuyer ma réflexion sur une figure développée par Frédéric Dejean et Heidi Hoerning (2010) qui montre les différentes étapes dans la structuration de la communauté : à chaque étape correspond un type de lieu, démontrant que l'histoire de toute communauté religieuse s'accompagne d'une géographie spécifique (figure 2).

Figure 2 : Trajectoires résidentielles et institutionnalisation religieuse

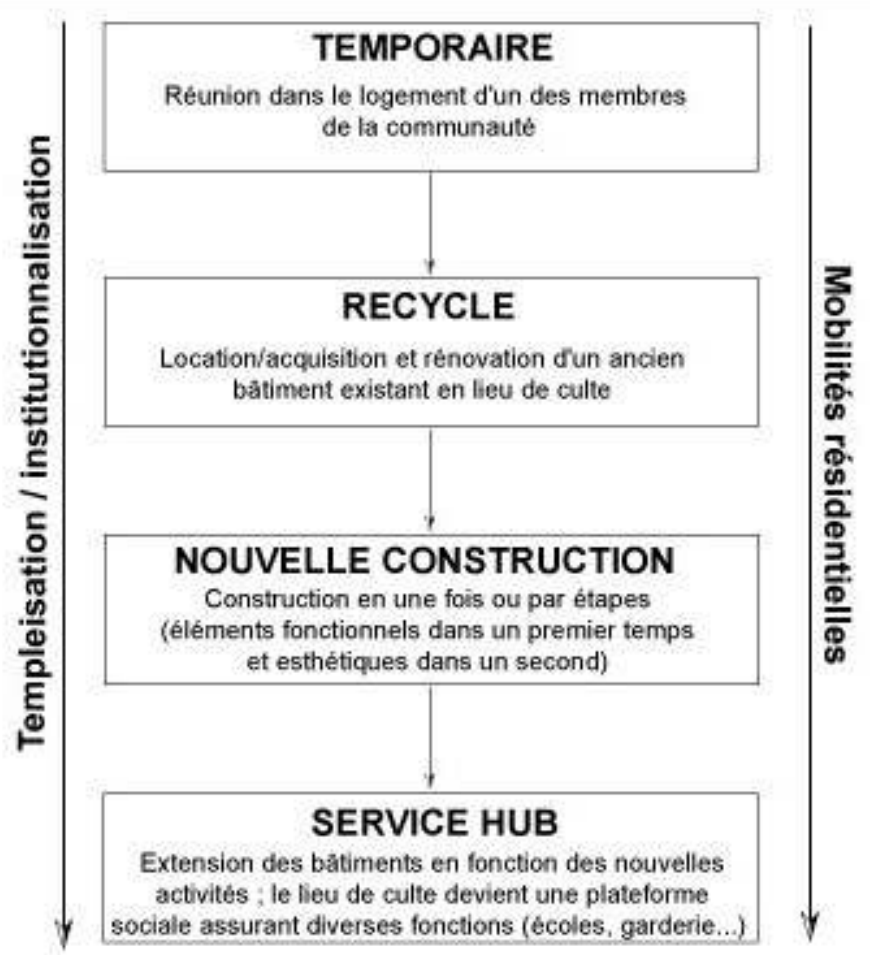

Commentaire accompagnant la figure 2 :

Dans le cas des études portant sur les manières dont les immigrés organisent leur religion dans leur pays d'accueil, plusieurs chercheurs ont montré qu'il y aurait aux Etats-Unis une tendance pour les institutions religieuses à assumer un processus de structuration similaire nommé « de facto congretionalism » (Cadge, 2008). A l'instar de Wendy Cadge, il semble nécessaire de nuancer ce processus. De prime abord, les temples tamouls en Île-de-France apparaissent remarquablement 
divers. Certains sont situés dans des bâtiments qui essaient de suivre les agama tandis que d'autres ont investis des lieux moins orthodoxes tels que des appartements ou des anciens ateliers (de mécanique et de menuiserie). Certains temples accueillent plusieurs prêtres et ont des cycles réguliers de rituels et de cérémonies, tandis que d'autres sont menés par un seul prêtre effectuant les rituels de manière ponctuelle. Ces variations sont liées à un large éventail de facteurs tels que l'âge du temple, l'éducation de ses fondateurs, le profil de ses visiteurs, la taille du temple, son implantation géographique, et les objectifs et les orientations (notamment politiques) de ou des personnes qui ont et continuent de jouer un rôle central dans l'organisation.

Ce modèle nous oblige cependant à penser la façon dont peuvent s'articuler les échelles de la sécularisation. Précisons aussi que nous entendons par sécularisation la diminution de la signification sociale de la religion par trois facteurs-clés de la modernisation (Willaime, 2006) : la différenciation sociale (qui fait que la religion n'est plus qu'une activité parmi d'autres et que ces autres activités sociales se développent selon leur logique propre entraînant une diversification des conceptions de la vie), la sociétalisation (qui représente le passage de la communauté à la société) et la rationalisation (qui a pour principal effet de changer la façon dont les gens pensent le monde et leur action). Cependant, la sécularisation ne signifie pas un déclin général de la religion. Il faut ici prendre en compte de quelles manières s'articulent les échelles de ce processus. C'est ainsi que Karel Dobbelaere (1981 : 11-12) distingue trois dimensions de la sécularisation : la laïcisation au niveau macrosocial (la place de la religion dans la société), le « changement religieux » au niveau mesosocial (les évolutions des organisations religieuses elles-mêmes) et l'implication religieuse à l'échelle individuelle (l'évolution des pratiques et attitudes individuelles). Si l'on prête bien attention à ces trois dimensions, on peut en effet très bien concevoir que la diminution de l'importance sociale de la religion, en particulier suite à la différenciation fonctionnelle des institutions et activités, n'implique pas en soi une diminution des pratiques et engagements individuels (Wilford, $2010: 337-338$ ). Dans cette perspective, le modèle de Dejean et Hoerning décrit ainsi une sorte de sécularisation qui laisse entrevoir la persistance d'archipels sacrés ${ }^{13}$ ou « sacred archipelagos » (Wilford, 2010).

11 Les changements de lieux participent pleinement de cette histoire. Bien entendu, la figure est un modèle. Cependant, elle permet de mettre en relation l'institutionnalisation des pratiques religieuses avec les trajectoires résidentielles, idéaltype de l'école de Chicago. Pour beaucoup d'auteurs (Ebaugh et Chafetz, 2000 ; Hirvi, 2010 ou encore Nesbitt, 2007), l'institutionnalisation de ces lieux de culte est d'une importance clé dans la production d'une communauté diasporique, car elle permet de négocier et favoriser émotionnellement et physiquement l'établissement des nouveaux arrivants en leur fournissant des ressources locales et en leur permettant la production d'un capital social indispensable. De ce point de vue, le lieu de culte joue le rôle d'un sas entre l'espace quitté et l'espace d'accueil dans lequel il faut acquérir des repères. Comme l'a noté Vertovec (1992) au sujet des hindous en Grande-Bretagne, la migration transforme aussi la signification de ces lieux, dans la mesure où, par rapport à ce qui se déroule à Sri Lanka, la vie paroissiale et la participation des membres de la 
communauté est plus intense. Par ailleurs, alors que les lieux de culte reflètent l'intention de l'enracinement de la communauté dans sa nouvelle place, ils permettent également de concevoir une nouvelle géographie de l'appartenance forgée sur ce que Vertovec (1997) nomme la "relation triadique» des sujets déplacés avec le lieu d'installation, le pays d'origine (homeland) et les autres polarités de la diaspora. De sorte que la transnationalisation entraîne le re-façonnement des paysages religieux. Dans le cadre de l'hindouisme en Île-de-France, une question se pose : comment pratiquer une religion $^{14}$ qui est fortement territorialisée ? En effet, l'idéologie des nationalistes hindous qui s'est développée au XIX siècle repose sur l'idée que l'Inde est exclusivement hindoue : le paysage indien est identifié au corps d'une déesse hindoue (Bharat Mata, la "Mère Inde»), ce qu'attestent et construisent les pèlerinages qui en relient les différents points et ce qui a pour corollaire que l'on ne peut être hindou qu'en Inde (Claveyrolas, 2008 et Trouillet 2012). Ceci pose à la fois la question du degré d'authenticité de l'hindouisme à Sri Lanka, mais plus grave encore celle de l'identité hindoue des Tamouls franciliens. D'autant plus que si l'on se réfère aux discours des indianistes, ces derniers voient une consubstantialité entre l'identité hindoue et l'appartenance de caste, la traversée des eaux figurant la transgression d'un interdit et d'un déclassement (Clémentin-Ojha, 2016). En effet, pour certains auteurs (SaglioYatzimirsky, 2015), le trauma de certains réfugiés sri-lankais résiderait dans le fait même d'avoir traversé les eaux noires appelées également kalapani ${ }^{15}$. Néanmoins, comme le montrent si judicieusement Bava et Capone $(2010: 8):$ « si les migrants utilisent souvent la religion pour affirmer leur appartenance à plusieurs communautés, ils l'utilisent aussi pour créer des 'géographies religieuses' alternatives, qui se superposent ou dépassent les frontières politiques nationales. Ces paysages religieux ne sont pas nécessairement des créations ex nihilo, mais souvent des adaptations au nouveau contexte qui permettent aux migrants de reproduire des pratiques issues de leur contexte d'origine». Ainsi, la déterritorialisation ne va pas sans le processus inverse de reterritorialisation. Bien entendu ce processus suggère des adaptations, des négociations et surtout de nouveaux discours sur les origines.

12 Je voudrais toutefois préciser que cette reconstruction de l'histoire de l'institutionnalisation des kovils en Île-de-France que je m'apprête à établir ne doit pas faire oublier le fait que la religiosité et les rites hindous sont essentiellement pratiqués à la maison, autour de l'autel domestique. En termes très généraux et en mettant l'accent sur des catégories spatiales, au sein du champ des rituels hindous, il y a une distinction importante entre les rituels domestiques (les rites dits grihya) et les rituels publics (dits shrauta) prenant place au sein ou à l'extérieur du kovil (par exemple : puja collective, abhisheka ou consécration de la murti, prasada). Néanmoins, selon mes observations, on assiste de façon croissante, à une survalorisation du temple. C'est au temple que l'on célèbre les fêtes et que se déroulent les principaux rituels biographiques - ou samskara (ces rites, initialement domestiques, marquent toutes les étapes de la vie des hindous mais désormais c'est au temple que s'effectue l'initiation à l'hindouisme, et plus généralement que les membres de la communauté apprennent à devenir un hindou). Ce processus de templeisation bouleverse l'équilibre entre la sphère domestique et le temple. Progressivement, les hommes et les prêtres acquièrent un rôle plus important que les femmes et les mères, qui sont traditionnellement celles qui initient les enfants à l'hindouisme. 
Jusqu'à récemment, seul un pourcentage relativement faible de la population française savait où et comment les Tamouls hindous pratiquaient leur foi parce que les institutions religieuses étaient pratiquement inexistantes lorsque les premiers immigrants sont arrivés.

« Au départ, il n'y avait pas de temple. Ce n'était pas important. Nous n'étions pas nombreux et on priait à la maison. Mais au fur et à mesure des arrivées, il fallait structurer la communauté, lui donner des repères. Spécialement pour la nouvelle génération. Moi je suis arrivé en 1980 à l'âge de 19 ans, je connais l'hindouisme. Mais ceux qui naissent en France, il leur faut un lieu pour pratiquer ...» (Extrait d'un entretien mené à la Chapelle en 2007).

De la fin des années 1970 jusqu'au début des années 1980, les chambres et les appartements des $18^{\text {ème }}$ et $10^{\text {ème }}$ arrondissements ont été utilisés pour la célébration de certaines fêtes. Ces lieux de culte étaient par définition temporaires mais reflétaient une nécessité : celle d'avoir un lieu pour la pratique religieuse. Le premier lieu de culte a été ouvert en 1985. Depuis les années 1990, dans plusieurs villes, les Hindous d'île-deFrance ont créé des organisations religieuses sous le statut associatif. On recense désormais 14 temples hindous en Île-de-France dont 11 possédés par des Sri Lankais, tous dans des bâtiments qui étaient initialement dévoués à d'autres activités.

Figure 3 : Temples hindous en Île-de-France

\begin{tabular}{|l|l|l|}
\hline Nom du temple & Localisation & Divinité \\
\hline Adhiparasakthi Kovil & Aubervilliers & (Mâry)Ammâ \\
\hline Sri Ayyapan Kovil & La Plaine Saint-Denis & Ayyapa \\
\hline Muthumari Amman Temple & Paris & (Mâry)Ammâ \\
\hline Sabariyassam Manchamatha temple & La Courneuve & Ayyapa \\
\hline Sathya Naarayana Padhuka Temple & Ivry/Seine & Shiva \\
\hline Sivan Parvathi Association & La Courneuve & Shiva \\
\hline Sri Ash Tala Lakshmi Derasthanam & Choisy-Le-Roi & Vishnu \\
\hline Sri Manicka Vinayakar Alayam & Paris & Ganesh \\
\hline Sri Muthukumaraswamy Alayam & Paris & Muruga(n) \\
\hline Sri Shity Vinayagar Temple & La Courneuve & Ganesha \\
\hline Sundara Vinayagar & Garges-lès-Gonesse & Ganesha \\
\hline
\end{tabular}

En effet, les Sri Lankais ont progressivement élu des comités de temple et collecté de l'argent pour louer ou même acquérir des anciens bâtiments pour les transformer en lieu de culte (anciennes menuiseries, ateliers de carrosserie ou de mécanique, agence de voyages...). L'octroi de l'autorisation par la préfecture pour recycler un lieu en kovil 
est parfois un processus long et difficile, comme c'est le cas pour le temple dédié à Murugan dans le 19 ème arrondissement :

«Le temple a été fondé et ouvert au public en octobre 1994. Cela a pris du temps pour l'ouvrir. Mais il fallait qu'on le fasse. C'est mon père qui est le fondateur de ce temple dédié à Murugan. À Keerimalay, dans le Nord Sri Lanka, mon père avait déjà fondé un temple dédié à Murugan, c'est donc naturellement, qu'arrivé en France, il s'est entrepris à la création d'un édifice similaire. Avant, ici c'était une agence de voyages. Pour trouver le local cela a pris énormément de temps. Dans les années 1990, quand on disait que l'on voulait louer un local pour des activités religieuses, les gens avaient peur. Il y avait beaucoup de problèmes avec la scientologie. On nous prenait pour une secte. De fait les gens étaient très suspicieux. On a donc créé une association, "Sri Muthukumaraswamy Alayam» et déposé une demande d'autorisation d'ouverture à la préfecture de Paris. Tous les Tamouls qui veulent ouvrir un temple opèrent de la même manière. Une fois l'autorisation acquise et le bâtiment trouvé, il faut faire venir les dieux. On ne peut pas vivre dans une ville où il n'y a pas de dieux. On a d'abord fait fabriquer à Sri Lanka trois murtis : une pour Ganesh, une pour Murugan et la dernière pour Aman. Les autels en bois, c'est nous qui les avons fabriqué. On se débrouille quand même. Mais le temple a été aménagé par un stapathi [maître architecte chargé de l'érection et de la décoration des temples hindous]. On l'a fait venir de Londres. C'est un Tamoul de Sri Lanka lui aussi. Il vient de Londres car c'est plus facile que de le faire venir de Sri Lanka, pour des raisons de visa. D'ailleurs c'est aussi à lui que les autres temples font appel lorsqu'ils veulent faire de nouveaux aménagements. L'ensemble de ces installations nous a coûté 70000 francs. C'est pour cela que nous avons construit les autels nousmêmes. Après on a fait une cérémonie de 9 jours pour que les divinités prennent corps dans les statues" (entretien mené avec Balakrishnakurukal, temple de Murugan, 2008).

Précisons que les kovils ne reflètent pas seulement la façon dont les hindous se perçoivent dans le nouveau contexte, mais ils deviennent activement les sites où la perception de l'hindouisme se forge pour les non-hindous. L'établissement de kovils a certes permis aux hindous de pratiquer leur religion, mais ces lieux agissent également comme un moyen de manifester et de localiser une tradition religieuse " étrangère " au sein de la vie culturelle française. Les kovils, représentent pour les Sri Lankais des lieux symboliques en ce qu'ils sont des " constructions rhétoriques destinées à désigner par connotation le territoire et la collectivité sociale qui l'érige " (Debarbieux, 1995 : 99). Les kovils sont des lieux saints qui peuvent se définir comme se situant à l'intersection de deux dimensions : l'une est verticale et permet de situer le rapport des hommes à l'espace terrestre (il s'agit en somme d'une inscription transcendantale au monde), l'autre est horizontale : il s'agit de l'inscription du lieu dans des réseaux sociaux plus ou moins denses, plus ou moins territorialisés, c'est-à-dire des organisations sociales fondées sur la caste, la parenté et la solidarité familiale permettant de relier les deux entre eux et la formation d'un présent étendu (Goreau-Ponceaud, 2008 : 262-263).

Les kovils rencontrent aussi le « langage de la normalisation » des autorités françaises qui peuvent les percevoir comme antinomiques plutôt que complémentaires au paysage existant (Peach et Gale 2003). Dans ce contexte, il n'y a pour le moment aucune création de temple qui soit comparable à celle de Sri Kamadchi Ampal à Uentrop près de Hamm (en Westphalie). Ce temple qui s'étend sur une parcelle de $700 \mathrm{~m}^{2}$ est largement influencé par l'architecture dravidienne: le gopuram fait $17 \mathrm{~m}$ de hauteur. Plus globalement, en France, l'étape 3 de la figure 3 n'a pas été réalisée. Même si la nostalgie joue un rôle indéniable dans l'implantation du lieu de culte, les kovils tamouls qui se 
sont progressivement constitués durant les décennies 1980 et 1990 concilient visibilité communautaire et respect des normes urbaines (figure 4 et 5).

Figure 4. Gopuram du temple de Shiva, Villianour (territoire de Pondichéry)

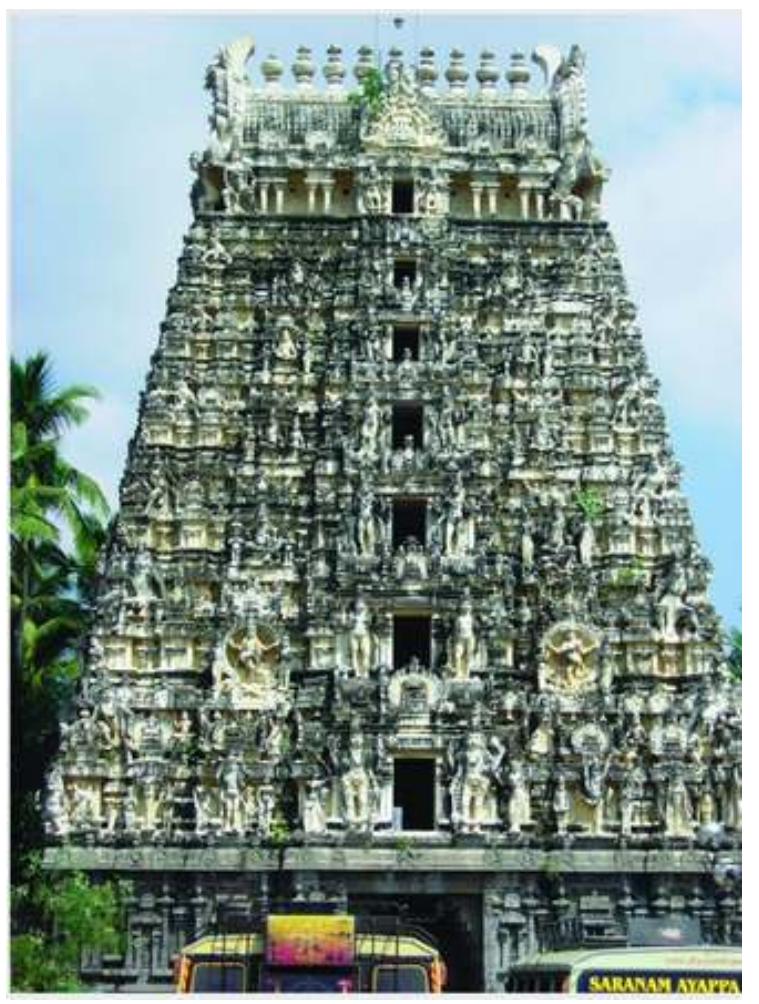

Crédit : auteur 
Figure 5. Gopuram du temple de Shiva, La Courneuve (Seine Saint-Denis)

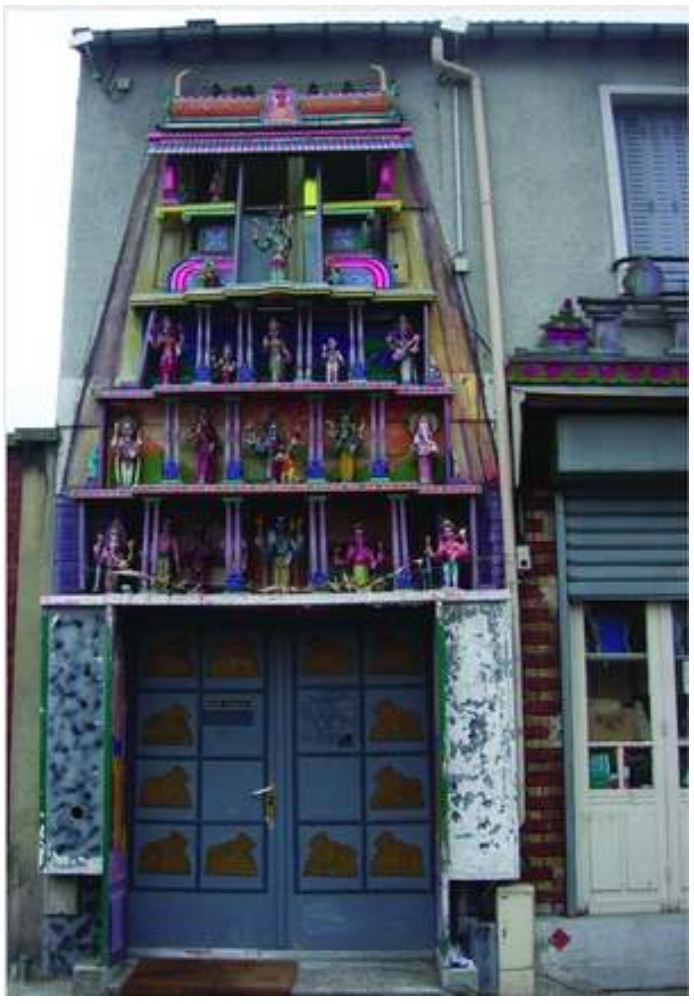

Crédit : auteur

Figure 6. Le Kovil
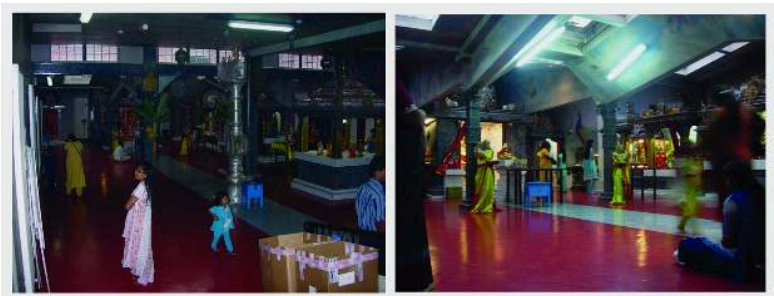

Photo 1-Vue générale. Les panneaux à gauche Photo 2- Au centre du temple, la mûrti de Shiva indiquent les horaires et les jours des prochaines (derrière la boitte à donations en bleu) cérémonies
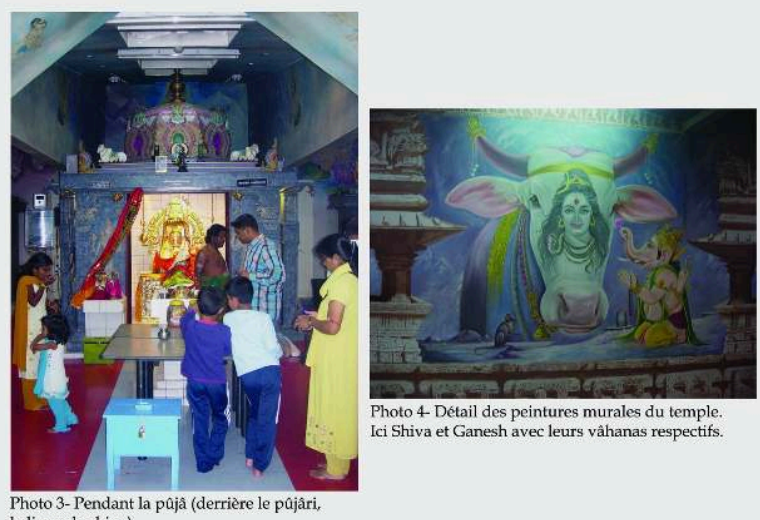

Photo 4- Détail des peintures murales du temple. Photo 3-Pendant la pajấ (derrière le pojjâri, le linga de shiva)

Crédit : auteur 
L'institutionnalisation des lieux de culte est souvent prise en charge par l'activation de réseaux transnationaux à travers lesquels le financement, les compétences techniques, les conseils d'architecture ou de savoir rituel sont transférés d'un endroit à l'autre. Il faut souligner ici le rôle indispensable du stapathi - ces spécialistes de vastu (traités en sanskrit sur l'art, l'architecture ou un artisanat, donnant les mesures, rapports et proportions que doivent avoir les constructions religieuses et les images divines en sculpture comme en peinture) qui déterminent l'emplacement des autels et des murtis. Pour la planification, la conception et le fonctionnement du kovil (figure 6), on enregistre, en provenance de l'Inde et Sri Lanka, l'émigration d'artisans qualifiés (sculpteurs, architectes) et de prêtres (ayer) chargés de les faire fonctionner. C'est ainsi que l'on retrouve dans la grande majorité des temples d'île-de-France, des prêtres bramâmes du Sud de l'Inde, venant pour la plupart de Rameswaram. Ces prêtres sont là pour une durée déterminée, généralement pour deux, voire trois années au maximum. Ainsi, les éléments nécessaires à la renaissance de l'hindouisme local, puisent leur substance dans un espace mondial, celui de la diaspora. On assiste ainsi à la mondialisation de l'hindouisme, religion longtemps fortement territorialisée.

Figure 7. Faire lieu c'est faire communauté

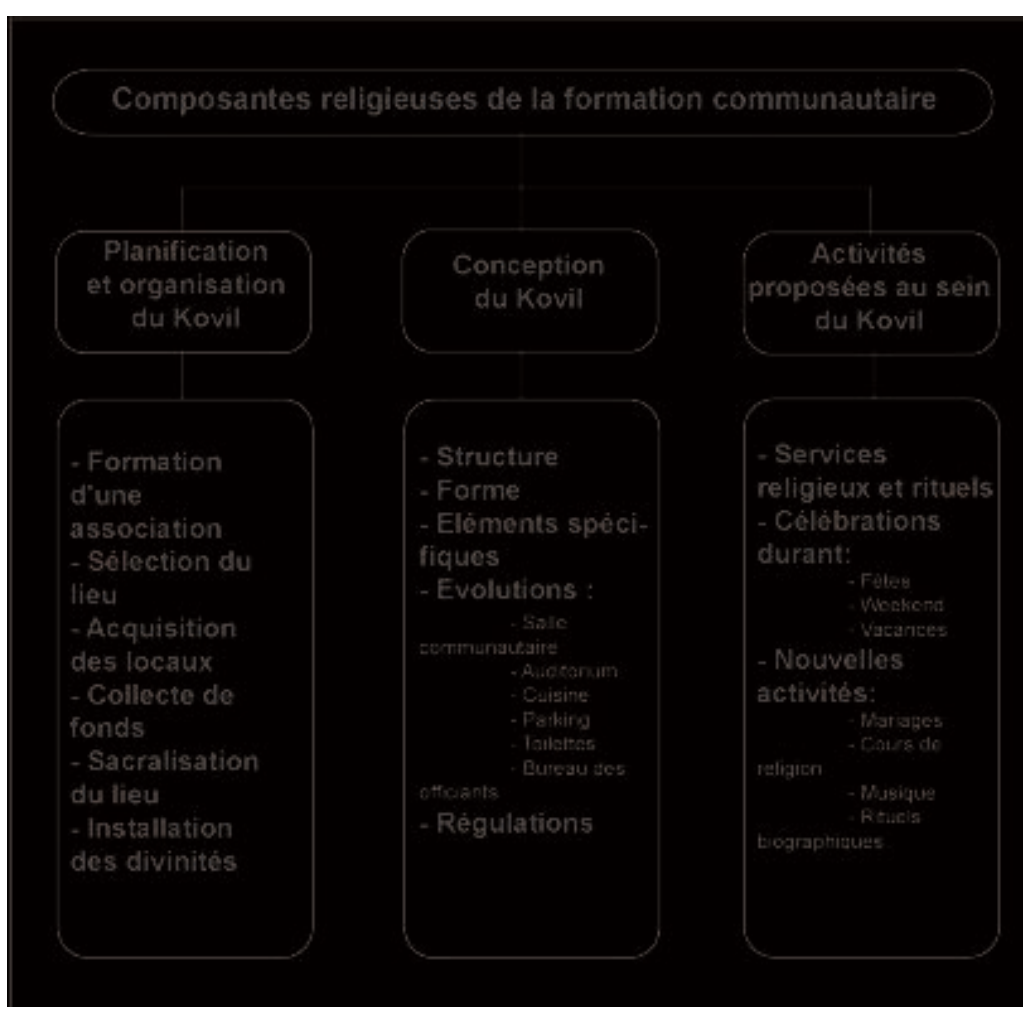

Source : auteur

19 Progressivement le lieu de culte devient ce que David Ley (2008) nomme un «service hub ", un centre fonctionnel qui devient également le centre de la vie sociale des individus, les services proposés concernant tous les domaines de la vie quotidienne (cours d'alphabétisation, de soutien, de danse...). D'autant plus que ce processus de templeisation, qui s'inscrit dans des circuits et des flux transnationaux, est renforcé par les associations nationalistes sri lankaises, très présentes dans les comités de temple. Ces associations ont construit une identité tamoule sur une base mythifiée de la 
"culture tamoule ancienne", qui est censée avoir existé avant la colonisation cinghalaise (Schalk, 1997 : 37). Les ingrédients de cette culture tamoule ancienne sont: la langue, la danse, la musique et la chanson, respectivement, bharathanatyam, veenai, miruthangam et sangeetham - les mêmes pratiques que les enfants apprennent au sein des temples. Les lieux de culte ont donc une dimension plurifonctionnelle, les plus spacieux, situés en banlieue, offrant toute une panoplie de services qui permettent de fournir un soutien dans le processus d'intégration. Ce processus de templeisation montre que la transplantation religieuse est toujours une transformation: la forte augmentation du nombre de réfugiés et l'arrivée des femmes et des enfants a joué un rôle décisif dans l'établissement et l'agrandissement des lieux de culte. De plus, la création d'un kovil permet la célébration de fêtes annuelles de la divinité associée au temple ; la plus importante étant le festival de Ganesh ou Ganesh Caturthi.

\section{Le festival de Ganesh}

Parmi tous ces temples, le temple Sri Manicka Vinayakar Alayam (dédié à Ganesh), fondé en 1985, s'affiche comme le "temple hindou de Paris» du fait de sa stratégie promotionnelle, et parce qu'il célèbre toutes les fêtes du calendrier hindou et en particulier qu'il organise, depuis 1996, le festival de Ganesh ou Ganesh Chaturthi. À cette occasion, les hindous d'Île-de-France organisent un défilé où le char du dieu à tête d'éléphant est promené dans les rues voisines du temple, situé au 17 rue Pajol. Au cours du trajet, le cortège, composé de danseuses et danseurs portant sur leurs épaules des arceaux de plumes de paon (cavadee ou kavadi) et sur leur tête des pots de terre cuite, passe devant des tas de noix de coco qui sont alors brisées et distribuées ${ }^{16}$. Viennent ensuite le char de Ganesh et de Murugan. Cette fête a connu plusieurs reformulations. C'est suite à un miracle qu'une fête en l'honneur de ce dieu est organisée tous les ans dans les rues de Paris. La date de la célébration, fixée en Inde le $4{ }^{\text {ème }}$ jour du mois lunaire de Bhadrapada, varie entre les mois d'août et septembre. A Paris, la fête est célébrée le dimanche le plus proche de la date du calendrier hindou, puisqu'elle nécessite de fermer momentanément à la circulation le quartier où se déroule la procession. La fête de Ganesh est une fête indienne célébrée dans toute l'Inde ; elle est antérieure au miracle et s'inscrit dans l'histoire coloniale de l'Inde et plus spécifiquement dans la lutte contre l'impérialisme britannique. Bal Gangadhar Tilak est à l'initiative de cette fête qui se déroula pour la première fois à Pune en 1893 (il y a des doutes concernant la date car certains partis politiques accordent la paternité de cette fête à Bhausaheb Javale). Il s'agissait pour cet indépendantiste de trouver un moyen de rassembler la population indienne par-delà les castes et les classes et de constituer un mouvement identitaire et national de masse. La réappropriation de cette fête par les tamouls en diaspora est significative et illustre une fois de plus l'hypothèse de la translocalité.

21 La reformulation de cette fête en diaspora mérite quelques explications: le 21 septembre 1995, en Inde comme dans l'ensemble des pays du monde où résident des Indiens, les murtis de Ganesh, de Shiva et les divinités lui étant associée ont bu le lait qui leur avait été offert en oblation. Le miracle du lait est une illustration supplémentaire $\mathrm{du}$ fonctionnement transnational de l'hindouisme. Cette diaspora tamoule, désormais connectée aux NTIC, a forgé "the age of instant miracle " (The Guardian, 23 septembre 1995). Comme le précise Vidal (1997: 884), « Le miracle put être constaté partout où se trouvaient les statues de Ganesh et des divinités associées à 
Shiva : que ce soit dans les temples ou chez les particuliers et aussi bien en Inde que dans le monde entier; partout, en fait, où il y avait des dévots ou même des simples curieux qui voulaient en faire l'expérience ". Ce phénomène ne dura que le temps d'une journée, mais a suffi à bâtir du côté du référent-origine une historiographie de la diaspora. L'arrivée du dieu Ganesh constitue ainsi une saisissante interrogation sur l'urbanisme contemporain, dans sa confrontation du global au local, d'autant qu'elle est susceptible de participer à la requalification urbaine. En combinant procession (mouvement) et stationnements (l'arrêt significatif devant les marchands les plus influents de la communauté, qui est l'endroit où les noix de coco sont brisées), cet événement a un caractère performatif. La mobilité, inhérente au religieux ne s'opère pas seulement hors des lieux de culte : l'espace du temple est structuré par la mobilité et sa fréquentation au quotidien, que ce soit par la pratique de la circumambulation autour de son enceinte ou par le rassemblement d'individus engendré à l'occasion d'un évènement exceptionnel du calendrier rituel (Chevrier, 2016; Claveyrolas et Delage, 2016).

L'itinéraire du défilé, inchangé depuis 1996, correspond à la centralité minoritaire de la Chapelle et à des zones résidentielles où se concentraient beaucoup de réfugiés tamouls dans les premières années de la migration (figure 2) de sorte que le temple est au cœur de ce que Berti et Tarabout nomment une juridiction divine ou Ksetra, c'est-à-dire une dimension du territoire inséparable de l'exercice du pouvoir politique $(2010: 13,20)$. La centralité de ce territoire est assuré par l'image divine et ses frontières (ou limites) sont construites et incorporées lors de la procession (puisque ce sont les corps qui expriment la matérialité de cette frontière). «Ce territoire-ksetra correspond autant à la définition hindoue du territoire sacré qu'aux réalités observables sur le terrain. Il témoigne d'un enracinement local du divin, c'est-à-dire d'une relation dynamique entre le particulier et l'universel» (Claveyrolas et Delage, 2016: 14). Le trajet processionnel parcourt le réseau des rues parisiennes dans la production adaptée d'un itinéraire ritualisé. Le déroulement de la procession est protégé par la police qui a un rôle, involontaire de légitimation de la fête (en Inde, l'ouverture d'une fête importante, hindoue ou catholique, impose toujours la présence d'instances civiles). En ce sens, le festival de Ganesh est "un rituel public qui renvoie à l'idée d'une délimitation sanctifiante de l'espace et du temps. Espaces et temps sont ainsi socialisés à travers des pratiques délibérées et de représentations locales qui s'accompagnent d'émotions à travers lesquelles l'expérience sociale s'éprouve, s'affiche, s'exprime et se débat" (Assayag, 2001, 261). La rue apparaît à ce moment-là comme une scène primitive du politique où les appartenances et les identités sont mises en contact et en tension les unes avec les autres. Cette tension exprime d'une certaine manière ce que Willaime nomme l'ultramodernité, c'est-à-dire la radicalisation de la sécularisation (2009: 204) dans laquelle il devient possible de prendre explicitement en compte, sans renier la laïcité, les apports spirituels, sociaux et culturels des religions. Les dominations spirituelles seront toujours en tension ou en conflit avec les dominations politiques. Comme le rappelle Willaime, ces tensions ou conflits «protègent la société tout aussi bien contre le risque de la domination spirituelle du politique que contre le risque de la domination temporelle» $(2009: 216)$.

23 A travers cette fête se pose à nouveaux frais la question de la visibilité de l'hindouisme et du contact entre une population citadine occidentale, marquée par l'impact de la sécularisation, et des populations immigrées où la religion infuse toute la réalité quotidienne. Rappelons cependant qu'il ne s'agit pas de déconnecter le religieux des 
évidences sociales. En d'autres termes, c'est l'Etat qui est laïque, pas la société. L'Etat central étant de moins en moins important en France, le politique s'ouvre de plus en plus volontiers au social (ce processus est d'ailleurs bien mis en évidence par François Ascher), et multiplie, de là, les zones de contact avec les Eglises et les croyances.

D'abord discrètes, ces implantations et ces manifestations religieuses tendent à s'afficher sur la base d'une relecture des séparations public/privé, sacré/profane, religieux/séculier. Et, comme une réponse à cette procession, les Pondichériens ont créé un espace Tamoul indien séparé au sein de l'Eglise catholique dans différentes villes de la région Île-de-France et ont institutionnalisé la première grande procession catholique tamoule indienne en 1999 : la procession de Notre-Dame de Velankanny. Il faut dire que l'arrivée des Tamouls de Sri Lanka a produit des transformations souvent perçues par les Tamouls de Pondichéry comme l'intrusion d'un possible désordre au cœur d'un paysage social et culturel qu'ils regardaient jusque-là comme homogène ou plutôt de façon sereine. En effet, ils se sont vus progressivement assimilés à ces «nouveaux entrants" arrivés selon eux de manière illicite, bousculant ainsi leur appartenance à la catégorie de vieux nationaux (Bastenier, 2004).

\section{Conclusion}

La religion qui a migré, qui s'est dé-localisée et mis en péril, au fil du temps se développe en une religion re-localisée et re-construite. Cette reconstruction possible par l'activation de flux transnationaux prenant corps et place dans un œcoumène diasporique s'est notamment manifestée dans le cas de l'hindouisme tamoul par un processus de templeisation apportant dans son sillage de multiples transformations. On note en effet les éléments d'évolution suivants : une visibilité accrue de l'hindouisme, un déséquilibre entre rites domestiques et rites publics, un bouleversement du rôle des femmes et des mères dans la transmission de l'hindouisme et surtout un brouillage des frontières profane/sacré, privé/public (particulièrement important pour les vieux nationaux).

Le festival de Ganesh n'est pas seulement un moyen, pour les Tamouls sri lankais, de rester en contact les uns avec les autres; ce festival doit aussi être compris comme un moyen de propager un projet diasporique, une conscience diasporique. Avec cette procession qui combine trois postures - le parcours, la mise en scène et le franchissement de limites sociales et spatiales - les Tamouls de Sri Lanka peuvent éprouver un sentiment identitaire, faire communauté. Cette construction communautaire n'est d'ailleurs aucunement dénoncée comme communautarisme par le gouvernement, les médias ou encore l'opinion publique française. Au contraire, il semble que du fait de son statut spécial dans l'imaginaire occidental - en lien avec l'orientalisme - l'hindouisme jouit en France d'une certaine attractivité. D'ailleurs, « les frontières entre les non-religieux et les religieux tendent quelque peu à s'estomper, ces derniers se sécularisent tandis que les premiers se spiritualisent" (Willaime, 2006: 775). Ce festival permet ainsi de ré-ancrer la religion qui s'est déterritorialisée et produire du religieux autrement. En d'autres termes, la prise de conscience de la multilocalisation stimule également la nécessité de se connecter conceptuellement avec d'autres, à la fois « ici » et « là ", qui partagent les mêmes « itinéraires » et les mêmes « racines ». Ce festival est tout aussi un moyen pour les Tamouls de revendiquer leur 
propre place dans la pluralité religieuse française qu'il est un indice d'une reconfiguration globale du religieux.

\section{BIBLIOGRAPHIE}

Aly-Marecar-Vinay N ; Vuddamalay V. (2007) « Les Indiens du Vietnam : un rôle pionnier », Hommes et Migrations, $n^{\circ} 1268-1269$, pp. 34-39.

Ansari H. (2002) 'The Woking Mosque: A Case Study of Muslim Engagement with British Society since 1889', Immigrants \& Minorities, 21(3), pp. 1-24.

Appadurai A. (1995), “The production of locality”, in Fradon R., Managing the diversity of knowledge, New York, Routledge, pp. 208-228.

Ascher F. (2007), La société évolue, la politique aussi, Paris, Odile Jacob, 310p.

Assayag J. (2001), L’Inde. Désir de nation, Paris : Odile Jacob, 347p.

Bastenier A. (2004), Qu'est-ce qu'une société ethnique ? Ethnicité et racisme dans les sociétés européennes d'immigration, Paris, PUF, 346p.

Bauberot J. (2004), Laïcité 1905-2005, entre passion et raison, Paris, Seuil, 288p.

Bauman M. (2009) “Templeisation: Continuity and Change of Hindu Traditions in Diaspora”, Journal of religion in Europe, 2, pp. 149-179.

Bava S ; Capone S. (2010) « Religions transnationales et migrations : regards croisés sur un champ en mouvement », Autrepart, vol. 4, n 56, pp. 3-15.

Berti D; Tarabout G. (2009), « Introduction », in Berti D., Tarabout G., Territory, Soil and Society in South Asia, Delhi, Manohar, pp. 9-38

Brubaker R. (2005) « The 'diaspora' diaspora », Ethnic and Racial Studies, n 28, pp. 1-19.

Bruland S. (2013) "Transgressing Religious Boundaries: The Power of Aesthetics in Tamil Catholic and Hindu Worship", Material Religion, 9 (4), pp. 418- 440.

Cadge W. (2008) "De Facto Congretionalism and the Religious Organizations of Post-1965 Immigrants to the United States: a Revised Approach", Journal of the American Academy of Religion, vol. 76, $\mathrm{n}^{\circ}$ 2, pp. 344-374.

Chevrier M-H (2016), Pratiques et valeurs spatiales, pèlerines et touristiques : grands et petits lieux de pèlerinage aujourd'hui, Thèse de géographie, Université de Lyon 2,470p.

Claveyrolas M ; Delage R. (2016), “Introduction”, in Claveyrolas M., Delage R., Territoires du religieux dans les mondes indiens. Parcourir, mettre en scène, franchir, Paris, EHESS, collection Purusartha, $\mathrm{n}^{\circ} 34$, pp. 11-43.

Claveyrolas M. (2008), “Les temples de Mère Inde, musées de la nation”, Gradhiva, n 7 nouvelle série, pp. 84-99. 
Clémentin-Ojha C. (2016), « Kālāpānī ou les limites à ne pas franchir. Le voyage en Angleterre du maharaja de Jaipur (1902) », in Claveyrolas M., Delage R., Territoires du religieux dans les mondes indiens. Parcourir, mettre en scène, franchir, Paris, EHESS, collection Purusartha, $\mathrm{n}^{\circ}$ 34, pp. 251-274.

Debarbieux B. (1995) «Le lieu, le territoire et trois figures de rhétorique », L'espace géographique, $\mathrm{n}^{\circ} 2$, pp. 97-112.

Dejean F ; Endelstein L. (2013) « Approches spatiales des faits religieux. Jalons épistémologiques et orientations contemporaines ", Carnets de géographes, $n^{\circ} 6$.

http://www.carnetsdegeographes.org/carnets_debats/debat_06_01_Dejean_Endelstein.php. 28/05/2017.

Dejean F ; Hoerning H. (2010), « Les nouveaux paysages religieux : regards croisés entre Paris, Montréal et Toronto », in Endelstein L., Fath S., et Mathieu S., Dieu change en ville, Paris, L'Harmattan, pp. 157-180.

DENIZEAU L. (2015) «L'infra et le mineur : du mode mineur de la réalité à l'anthropologie existentiale dans l'œuvre d'Albert Piette », Le Philosophoire, nº 44, pp. 177-199.

Dobbelaere K. (1981) « Secularization : a multidimensional concept », Current Sociology, $\mathrm{n}^{\circ} 29$, 3-153.

Ebaugh HR ; Chafetz JS. (2000), Religion and the Immigrants : Continuities and Adaptations in Immigrant Congregations, New York, Altamira Press, 492p.

Fourcade MB. (2008), « Reconstruire une petite Arménie au Québec. Réflexions autour d'un patrimoine diasporique », in Fourcade MB., Legrand, C., Patrimoines des migrations, migrations des patrimoines, Laval, PUL, pp. 17-34.

Gale R; Peach C. (2003) "Muslim, Hindus and Sikhs in the new religious landscape of England", Geographical Review, vol. 93, n 4, pp. 469-490.

Goreau-Ponceaud A. (2018) "La Chapelle : un quartier pour qui ?", Métropolitiques, en ligne : http://www.metropolitiques.eu/La-Chapelle-un-quartier-pour-qui.html

Goreau-Ponceaud A ; Gallo E. (2015) «L'immigration sud-asiatique : routes migratoires, parcours de santé et intégration », Migrations et société, vol. 27, n 161, pp. 49-74.

Goreau-Ponceaud A. (2014), "Ganesha Chaturthi and the Sri Lankan Tamil Diaspora in Paris: inventing strategies of visibility and legitimacy in a plural 'mono-cultural' society", in Gallo E., Migration and Religion in Europe. Comparative perspectives on South Asian experiences, Farnham: Ashgate, pp. 211-231.

Goreau-Ponceaud A. (2011), “Tamils in France”, in Irudaya R., Percot M., Dynamics of Indian Emigration: Historical and current perspectives, Delhi, Routledge, Chapter 3, pp. 64-90.

Goreau-Ponceaud A. (2008) La diaspora tamoule : trajectoires spatio-temporelles et inscriptions territoriales en Île-de-France, thèse de doctorat de l'Université de Bordeaux 3, 427p.

Grillo RD. (2010), “An excess of alterity? Debating difference in a multicultural society”, in Vertovec S., Anthropology of Migration and Multiculturalism, New-York and London, Routledge, pp. 19-38.

Hirvi L. (2010) 'The Sikh Gurdwara in Finland: Negotiating, Maintaining and Transmitting Immigrants' Identities', South Asian Diaspora, vol. 2, n² 2, pp. 291-32. 
Hovanessian M. (2007), «La notion de diaspora : la question de la temporalité », in Arméniens et Grecs en diaspora : approches comparatives, Actes du colloque européen et international organisé à l'Ecole française d'Athènes, Athènes, École française d'Athènes, 2007, p. 7-17.

Jacobsen KA. (2008), "Processions, public space and sacred space in the South Asian Diasporas in Norway", in Jacobsen KA., South Asian religions on displays. Religious processions in South Asia and in the Diaspora, London, Routledge, pp. 191-204.

Ley D. (2008) “The immigrant church as an urban service hub”, Urban Studies, vol. 45/10, pp. 2057-2074.

Mantovan G. (2015) « Ils étaient des rois... : l'adieu aux armes d'anciens combattants des Tigres tamouls exilés en France », Migrations Société, vol. 27, n 161, pp. 89-104.

Meyer E ; Madavan D. (2015) «Sri Lanka : les séquelles de la guerre », Hérodote, n 158, 2015, pp. 219-237.

Narayanan V. (1992), "Creating South Indian Hindu Experience in the United States," in Raymond BW., A Sacred Thread: Modern Transmission of Hindu Traditions in India and Abroad, Chambersburg, Anima Publications, pp. 147-176.

Nesbitt E. (2007), 'The Contribution of Nurture in a Sampradaya to Young British Hindus' understanding of their Tradition', in HINNELS JR., Religious Reconstruction in the South Asian Diasporas, New York, Palgrave, pp. 51-73.

Piette A. (1992) Le mode mineur de la réalité. Paradoxes et photographies en anthropologie, Louvain-la-Neuve : Peeters, $118 \mathrm{p}$.

Saglio-YATZIMIRSKY MC. (2015) “Kalapani : le trauma de la traversée dans la migration des demandeurs d'asile tamouls du Sri Lanka », Migrations société, vol. 27, n 161, pp. 75-87.

Sebastia B. (2002) "Mariyamman - Mariyamman. Catholic pratices and image of Virgin in Velankanni (Tamil Nadu)", Pondy Papers in Social Sciences, $n^{\circ} 27,73 p$.

Shalk P. (1997), « Resistance and martyrdom in the process of state formation of Tamililam", in Pettigrew, J., Martyrdom and Political Resistance, Amsterdam, Amsterdam University Press, pp. 61-83.

Slyomovics S (1995), “The Muslim World Day Parade, in Nation and Migration”, in Van Der Veer P., The politics of space in the South Asian Diaspora, Pennsylvania, University of Pennsylvania Press, pp. 157-177.

Trouillet P-Y (2012), « Overseas Temples and Tamil Migratory Space », South Asia Multidisciplinary Academic Journal [on line ], vol. 6, http://samaj.revues.org/3415

Vertovec S. (1997) “The Meanings of Diaspora as Exemplified by South Asian Religions”, Diaspora, vol. 6, n 3, pp. 277-300.

Vertovec S. (1992) “Community and Congregation in London Hindu Temples: Divergent Trends", New Community, 18(2), pp. 251-264.

Vidal D. (1997) « Empirisme et croyance dans l'hindouisme contemporain : quand les dieux boivent du lait », Annales, vol52, $\mathrm{n}^{\circ} 4$, pp. 881-915.

Wilford J. (2009) « Sacred archipelagos: geographies of secularization », Progress in Human Geography, vol. 34, $\mathrm{n}^{\circ}$ 3, pp. 328-348.

Willaime JP. (2009) « Pour une sociologie transnationale de la laïcité dans l'ultra modernité contemporaine », Archives de sciences sociales des religions, $n^{\circ} 146$, pp. 201-218. 
Willaime JP. (2006) « La sécularisation : une exception européenne ? Retour sur un concept et sa discussion en sociologie des religions », Revue française de sociologie, vol. 47, n 4, pp. 755-783.

\section{NOTES}

1. . Les Tigres de libération de l'Eelam tamoul ou LTTE sont un mouvement indépendantiste, fondé en 1976 par Velupillai Prabhakaran, dont le but affiché est de défendre les Tamouls de Sri Lanka. Pour cela, l'organisation demande le droit à l'autodétermination et la création d'un Etat, le Tamil Eelam. La défaite militaire du 17 mai 2009 a ébranlé le mouvement.

2. . Dans cette contribution, le terme diaspora est utilisé comme une catégorie analytique permettant de décrire les processus de migration et les relations transnationales qui lient les Tamouls sri-lankais déplacés entre eux, avec le pays d'origine (ou homeland) et l'ailleurs (œcoumène diasporique). Plus exactement, dans la continuité des travaux de Brubaker il s'agit moins de se demander ce qu'est une diaspora, que d'étudier les sens du terme de diaspora donné par les Tamouls eux-mêmes qui se réclament d'une diaspora. Comme le précise Brubaker: "In sum, rather than speak of 'a diaspora' or 'the diaspora' as an entity, a bounded group, an ethnodemographic or ethnocultural fact, it may more fruitful, and certainly more precise, to speak of diasporic stances, projects, claims, idioms, practices, and so on" (2005:13).

3. Depuis le début des années 2000, les débats nationaux en France comme dans d'autres pays de l'Union Européenne (Pays-Bas, Allemagne et Grande-Bretagne en particulier) ont vu se développer un nouveau thème : celui de la crise, voire de l'échec des modèles d'intégration des migrants ; que ces modèles soient multiculturalistes ou républicains. La politisation du débat sur la crise et l'échec des modèles ont eu pour résultat de re-politiser très fortement la notion de modèle d'intégration. En France, cela a pris la forme d'un discours complexe sur la diversité et la République, particulièrement depuis les «émeutes des banlieues» de 2005. Plus largement, la constitution progressive d'une "forteresse Europe " s'est accompagnée par une réaffirmation, dans beaucoup d'États européens, d'attitudes racialisantes envers les migrants et les minorités, ces derniers incarnant une altérité indépassable face une identité européenne réifiée et essentialisée (Goreau-Ponceaud et Gallo, 2015).

4. . Malgré la nature problématique et contestée du terme "communauté », je l'utilise pour indiquer la construction symbolique, créée par la perception des limites par les membres d'un groupe. La notion réelle de communauté soutient souvent un sens fluide et pragmatique pour les individus, ce qui révèle qu'il s'agit d'une notion à plusieurs couches et multivalente qui ne signifie pas l'adhésion ou l'appartenance à un groupe étroitement défini. Chaque personne peut être membre de communautés différentes, peu définies et en évolution: les limites de la communauté ne sont pas fixes mais changeantes, situationnelles et perméables, sachant aussi que les communautés s'interpénètrent et se recoupent. Dans cette contribution, je parlerai également de communautés religieuses, dans le sens où chaque hindou voue généralement, soit par tradition familiale, soit par vocation individuelle, un culte à une divinité particulière, qu'il nomme sa « divinité d'élection » (iștadevatā). Ce culte (pūjā) qui était majoritairement domestique est de plus en plus pratiqué au temple décrivant progressivement des communautés religieuses.

5. . Plutôt que de territoires géographiques aux frontières établies, le territoire relationnel est ouvert, à la géographie instable, en recomposition et en redéploiement permanent. Les liens tissés entre les membres de la diaspora et la diversité des parcours migratoires composent seuls les contours de ce dernier, dans lequel il existe un certain équilibre entre bonding (cohésion sociale, liens très soudés) et bridging (connectivité sociale, création de passerelles), faisant ainsi coexister liens forts et liens faibles.

6. . Selon Laurent Denizeau, au fil de l'œuvre d'Albert Piette, le mode mineur de la réalité est devenu «le socle d'une anthropologie existentiale, tournée non plus vers les représentations 
sociales et culturelles qui conduisent à désincarner et abstraire le vécu humain mais vers notre manière spécifiquement humaine d'être au monde » $(2015: 178)$. Le mode mineur en insistant sur les écarts, les contrastes et les ambivalences dévoile ainsi la figure de l'homme partagé entre engagement dans des énoncés de sens et le dégagement de ceux-ci.

7. . Comme le montrent Sophie Bava et Stefania Capone «les processus de transnationalisation religieuse deviennent ainsi une clef d'analyse indispensable des réseaux migratoires, puisque l'impact des références et des espaces religieux dans la constitution ou la réorientation des trajectoires migratoires s'avère aujourd'hui particulièrement important » $(2010: 7)$.

8. . Velankanni est un centre de pèlerinage situé dans l'État indien du Tamil Nadu qui est très célèbre pour sa Vierge, appelée Arokkiya Mata ("Notre Dame de la Bonne Santé") ou Velankanni Mata. Son culte, initié par les Portugais au début du XVII siècle, s'est intensifié et jouit aujourd'hui d'un succès croissant, mesuré par les centaines de milliers de pèlerins de toutes les castes et croyances, et de tous les États d'Inde, qui assistent à son festival de dix jours du 29 août au 8 septembre. Dans son étude ethnographique du catholicisme à Velankanni Brigitte Sebastia, montre, d'une part, la forte influence de l'hindouisme sur le culte de la Vierge et le sanctuaire et, d'autre part, la portée fédérative du sanctuaire et de la Vierge, dont l'étendue est unique en Inde (Sebastia, 2002). Elle a également noté que le catholicisme à Velankanni se présente sous deux aspects radicalement opposés : 1/un aspect orthodoxe au sein de l'église symbolisé par une liturgie identique à celle célébrée en Occident et $2 /$ un aspect hétérodoxe à l'extérieur de l'église résultant d'un mélange subtile de formes rituelles ou dévotionnelles dérivées du catholicisme et de l'hindouisme.

9. Plus largement, la création de lieux de culte au sein des diasporas indiennes a reçu une attention considérable des sciences sociales dans les pays où la population d'origine sud-asiatique est numériquement importante ou tout au moins visible. En effet, divers auteurs ont reconnu combien les temples ou

les gurdwaras constituent des lieux importants pour les migrants dans la mise en place d'un sentiment communautaire permettant de reproduire un chez-soi déterritorialisé (Peach et Gale, 2003 et Ansari, 2002).

10. . Selon Claude Raffestin (1986), le territoire est une réordination de l'espace dont l'ordre est à chercher dans les systèmes informationnels dont dispose l'homme en tant qu'il appartient à une culture. Le territoire peut être considéré comme de l'espace informé par la sémiosphère. Les arrangements territoriaux constituent une sémiotisation de l'espace, espace progressivement "traduit" et transformé en territoire. L'écogenèse traduit ce processus.

11. . Le « nationalisme méthodologique » consiste à étudier les processus et les acteurs sociaux en considérant les frontières nationales comme l'échelle significative d'analyse et en suggérant un isomorphisme entre Etat, identité nationale et nation. Cette hypothèse repose sur la domination d'un système de représentation, la fixité. Le périmètre, la frontière donnent corps et naissance à l'Etat qui se manifesterait par deux propriétés ineffaçables: l'exclusivité et l'exhaustivité. Bousculer cette hypothèse c'est dépasser les dualités entre global et local, national et international, eux et nous. Notons également que c'est l'émergence de l'Etat-nation qui a entraîné une façon de redéfinir la place du religieux et du séculier.

12. Chez les Tamouls, le kōyil ou kovil ou encore koil désigne le temple hindou.

13. . Comme le note Justin Wilford, « a central question for geographers of religion, then, should be to what extent is the social differentiation that leads to 'sacred umbrellas' integrally related to a spatial differentiation that leads to what I term 'sacred archipelagos' ?” (2010: 337).

14. Précisons toutefois que l'hindouisme n'est pas structuré par des dogmes, ni par une Eglise institutionnelle et sa dénomination même est un terme exogène. En somme l'hindouisme n'a pas véritablement d'existence en tant que religion unifiée et c'est finalement, selon les indianistes, la structure en castes et l'unité familiale qui caractérisent la vie des hindous. 
15. . Dans la tradition des siècles derniers, les castes supérieures sont tenues de vivre sur la terre mère où peut s'accomplir le dharma. Or en traversant les eaux, il n'est plus possible de maintenir les lois de caste qui garantissent le statut, c'est-à-dire les règles de commensalité et de mariage (Saglio-Yatzimirsky, 2015). On peut nuancer cet argument en rappelant l'histoire particulière et récente qui a marqué les réfugiés sri-lankais, en particulier les ex-combattants. A ce sujet voir Mantovan, 2015.

16. A travers ces quelques lignes, je ne souhaite pas diffuser un regard exotique signifiant pour la population décrite l'enfermement dans la tradition, l'excluant de toute histoire, déterminant des pratiques qui ont un effet réel sur le monde réel, mais bien monter que la reconnaissance de la différence est devenue la condition même de la reconnaissance de cet universel par quoi nous sommes identiques (Baubérot, $2004: 128$ ).

\section{RÉSUMÉS}

Depuis la décennie 1980, les réfugiés en provenance de Sri Lanka arrivent de manière régulière en France et constituent le plus grand groupe hindou, accentuant la pluralisation du paysage religieux et culturel. Si la présence de la religion peut être discrète et dérobée aux regards, on observe, en lien avec la consolidation des chaînes migratoires tamoules, le retour en force des processions, à l'instar de la procession de Ganesh. En tant que tel, l'hindouisme ne représente pas seulement la tradition d'une minorité religieuse ; il met également en tension des acteurs déjà ancrés dans la ville et des populations migrantes nouvellement arrivées. C'est donc à travers l'articulation entre migration, religion et espace que je vais aborder l'enjeu du religieux, et plus spécifiquement l'enjeu du religieux en mode mineur en ville. Entre local (la rue, le quartier) et le global (flux d'immigration transnationaux), c'est l'enjeu du rapport à l'espace qui constitue l'axe de cette réflexion où l'espace sera considéré comme un medium des rapports sociaux qui permet d'interroger de façon originale des recompositions du fait religieux aujourd'hui

Since the 1980s, refugees from Sri Lanka have been living in France and make up the largest Hindu group. In recent years this migration, and more generally the South Asian migration, has radically transformed the French social landscape. In a context of dramatic growth in religious diversity, Hinduism represents not only a minority religious tradition but also a challenge to French laïcité. A new visibility/invisibility dialectical relation has also become a major issue. Although the presence of religion may be discreet and hidden, religious processions make a powerful comeback, such as Ganesh Caturthi organized by Sri Manicka vinayakar Alayam every year in the streets of Paris. I will tackle the changing dimension of religious questions in an urban environment through the articulation between immigration, religion and space. Between the local (the street, the "quartier») and the global (transnational migration nexus), I will analyze space as a medium of social connections which shed new light on the reconfiguration of religion. Through the study of the internal multiplicity of Tamil immigration and of the localization of places of worship, this paper will explore the development of Sri Lankan Hinduism in Paris and its metropolitan region. I will also show how Hindus have to negotiate their status and sometimes transform their practices to be accepted by the host state. 
INDEX

Thèmes : Carnets de recherches

Keywords : Hinduism, Tamils, Migration, Transnationalisation

Mots-clés : hindouisme, Tamoul, migration, transnationalisation

\section{AUTEUR}

ANTHONY GOREAU PONCEAUD

Géographe

Maitre de conférences

Université Bordeaux, LAM UMR 5115

anthonygoreau@yahoo.fr 